\title{
Molecular Rotors Image Intracellular Viscosity
}

\author{
Marina K. Kuimova* \\ Grammaticakis-Neumann Prize winner 2011
}

\begin{abstract}
This review describes a new method for quantitative measurement and spatial imaging of microviscosity within individual domains of live cells. The method is based on fluorescence detection from small synthetic molecules termed 'molecular rotors', which are characterised by a strong response of fluorescence lifetimes or spectra to the viscosity of their immediate environment. We have demonstrated that the quantitative determination of viscosity is possible using lifetime-based molecular rotors and ratiometric molecular rotors. The ratiometric imaging of viscosity benefits from a very fast signal acquisition. We have illustrated this advantage by monitoring changes in intracellular viscosity during photodynamic therapy, which is a clinically utilised modality for the treatment of neoplastic disease.
\end{abstract}

Keywords: FLIM · Fluorescence imaging · Molecular rotors · Photodynamic therapy · Viscosity

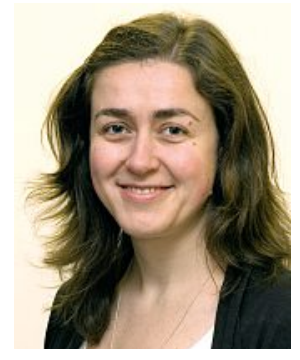

Dr. Marina Kuimova received a M.Sc. in Chemistry from Moscow State University in 2001, and a $\mathrm{PhD}$ from Nottingham University in 2005. Her $\mathrm{PhD}$ work was performed under supervision of Prof. Michael W. George. This was followed by a postdoctoral position at Imperial College London with Prof. David Phillips, working on spectroscopy and imaging related to two-photon excited photodynamic therapy (PDT) of cancer. In

\footnotetext{
${ }^{*}$ Correspondence: Dr. M. K. Kuimova

Chemistry Department, Imperial College London

Exhibition Road, South Kensington

SW7 2AZ, U.K.

Tel. +44 2075948558

Fax +44 2075945804

E-mail: m.kuimova@imperial.ac.uk
}

2007 Dr. Kuimova obtained an independent position as a Life Sciences Interface EPSRC Fellow at Imperial. Dr. Kuimova is currently the EPSRC Career Acceleration Fellow in the Department of Chemistry at Imperial College London.

Kuimova's current work uses variety of imaging and spectroscopic techniques to elucidate the nature of processes involved in cell function and death, including those during PDT treatment of cancer. From 2008 her group's focus was on developing synthetic fluorescent probes for microscopic viscosity termed 'molecular rotors'. Her research interests also include ultrafast processes involving excited states of biomolecules, such as DNA, and photophysics of coordination compounds. She has been awarded SET for Britain Westminster and Roscoe medals for early career researchers in sciences and engineering by the UK Parliament (2009), GrammaticakisNeumann Prize by the Swiss Chemical Society (2011) and the British Biophysical Society Young Investigator Award (2012).

\section{Introduction}

Viscosity is one of the major parameters determining the diffusion rate of species in condensed media. In the simplest case, viscosity governs the rate of bimolecular reactions, but, at the same time, it is also the characteristic that has important implications for mechanical properties of materials and their chemical stability. As such, viscosity is a major parameter of interest in engineering and industrial process design. On the other hand, viscos- ity plays an important role in biological systems, as it determines the rate of diffusion at the microscopic level within individual cell compartments. As the main determinant of diffusion rate within and across the domains of live cells, changes in viscosity of these domains must have important consequences for normal cellular function. It is therefore not surprising that abnormal levels of viscosity have been linked to disease and malfunction in animals and human subjects. ${ }^{[1]}$ A common example is abnormalities in viscosity of membranes, ${ }^{[1 \mathrm{a}, \mathrm{e}]}$ which were detected in patients with atherosclerosis, ${ }^{[1 b]}$ diabetes, ${ }^{[1 \mathrm{c}]}$ Alzheimer's disease ${ }^{[1 d]}$ and even in cell malignancy. ${ }^{[1 a]}$

Given the evidence of links between abnormal levels of viscosity and disease, measuring microviscosity in the individual domains of live cells is a worthwhile task. The main problem in this regard is one of scale: the proposed measurement method must sample the microscopic length scales, relevant to cellular studies. While mechanical methods to measure the bulk macroscopic viscosity are well developed, imaging local microscopic viscosity remains a challenge. A range of spectroscopic methods has been developed in the last few decades, which are capable of reporting on viscosity or diffusion in microscopic regions of space. These include single particle tracking, ${ }^{[2]}$ fluorescence recovery after photobleaching, ${ }^{[3]}$ steady state and time resolved fluorescence anisotropy, ${ }^{[4]}$ fluorescence correlation spectroscopy ${ }^{[5]}$ and monitoring the rates of the fast photochemical processes, e.g. quenching. ${ }^{[6]}$ In this review I will discuss an alternative 
approach which not only allows to measure microviscosity but also enables imaging of local microviscosity in individual compartments of live cells. This approach is based on fluorescence measurements of small synthetic molecules termed molecular rotors.

Molecular rotors are fluorescent molecules, in which the non-radiative decay of the fluorescent excited state is strongly influenced by the viscosity of the surrounding medium. ${ }^{[7,8]}$ Within this class of fluorophores intramolecular rotation or twisting dominates the photophysical properties of the dye, by changing the population balance between the 'bright' fluorescent excited state and the 'dark' state. The structural change, such a twisting, is typically viscosity dependent and leads to a population of a dark non-emissive excited state or activates other non-radiative decay pathways in a rotor. At the same time, the viscosity of the immediate environment of the rotor, be it homogeneous solution or heterogeneous mixture, governs the rate of the rotation or conformational change within the molecular frame. In a non-viscous environment, rotation is unobstructed and the population of the 'dark' state is efficient, leading to low fluorescence. Conversely, in a viscous environment the intramolecular rotation is slowed down and this causes the drastic change in the fluorescence parameters such as intensity, decay time, and sometimes even the spectral profile. The benefits of this approach are that changes in the fluorescence parameters with viscosity are gradual and can be described by mathematical equations, as will be discussed below in more detail. Thus, the responses of the rotor can be directly calibrated. Based on such calibration, viscosity can be directly measured by detecting the change in the fluorescence parameters (spectra or lifetimes) of molecular rotors.

Due to the ease of calibration, fast fluorescence acquisition times and excellent spatial resolution offered by fluorescence imaging, the molecular rotor approach has been applied to viscosity studies of biological fluids and fibres, ${ }^{[9]}$ as well as sol-gel and polymer samples ${ }^{[10]}$ and for imaging of flow patterns. ${ }^{[11]}$ This review is dedicated to our own investigations using fluorescent molecular rotors with the aim to develop accurate tools to measure in vivo and in vitro viscosity in a wide range of biological environments, including those present within a living cell.

\section{Förster-Hoffman Equation}

The dependence of fluorescence quantum yield $\left(\Phi_{f}\right)$ on viscosity $(\eta)$ is described by the Förster-Hoffman equation, ${ }^{[12]}$ Eqn. (1):

$$
\Phi_{f}=z \eta^{\alpha}
$$

where $z$ and $\alpha$ are constants.

It directly follows from this equation that a plot of $\log \Phi_{f}$ as a function of $\log$ $\eta$ should yield a straight line with a slope of $\alpha$. The later theoretical work has identified that $\alpha$ is expected to fall in the range between $1 / 3$ and $2 / 3$ and this was supported by experimental data.[13]

As was mentioned previously, it is expected in molecular rotors that the strong dependence of $\Phi_{f}$ on viscosity is the result of non-radiative processes being activated by intramolecular rotation. Radiative $\left(k_{r}\right)$ and non-radiative $\left(k_{n r}\right)$ decay rate constants can be calculated from the main parameters of molecular fluorescence, the fluorescence quantum yield $\left(\Phi_{f}\right)$ and the fluorescence lifetime $\left(\tau_{f}\right)$, as defined by Eqn. (2):

$$
\Phi_{f}=\frac{k_{r}}{k_{r}+k_{n r}}=k_{r} \tau_{f}
$$

Furthermore, Eqn. (2) can be easily substituted into Eqn. (1) to produce a modified Förster-Hoffman equation, Eqn. (3):

$$
\tau_{f}=\frac{z \eta^{\alpha}}{k_{r}}
$$

and the logarithmic form, Eqn. (4)

$$
\log \tau_{f}=\log \left(\frac{z}{k_{r}}\right)+\alpha \log \eta
$$

Thus a plot of $\log \tau_{f}$ as a function of $\log$ $\eta$ should yield a straight line with a slope of $\alpha$. The intercept of the line gives information about the radiative rate constant, $k_{r}$, for the molecule. The straight line serves as a calibration plot for the fluorescence response of the molecular rotor.

\section{Quantitative Measurements of Viscosity in Cells}

The Förster-Hoffman equation provides the framework for measuring viscosity in microscopic compartments of biological samples, using fluorescent molecular rotors. The cellular studies require the biocompatible molecular rotors to be available, which are characterized by high uptake to cellular compartments of interest and by low dark and photo-toxicity to cells. Several such rotors have been designed and their structures are shown in Fig. 1. For all of these molecules, it has been reported that $\Phi_{f}$ increases as a function of viscosity according to the FörsterHoffman equation.

Benzylidene malononitriles, e.g. commercially available 9-(2,2-dicyanovinyl) julolidine (DCVJ, Fig. 1a), are the most actively studied molecular rotors to date. These dyes display a marked increase in the fluorescence intensity as solvent viscosity increases, attributed to the existence of the low-energy twisted intramolecular charge transfer (TICT) state. The TICT state serves as a non fluorescent 'dark' state and is efficiently populated upon photoexcitation in non-viscous solvents. Utilising the change in $\Phi_{f}$ with viscosity in DCVJ, viscosity was measured in solvent mixtures and in solutions of dextran and starch, with viscosity ranging from 1 to $300 \mathrm{cP}$. ${ }^{7]}$

These bulk measurements relied on the known concentration of the fluorophore in a cuvette, which enabled the determination of the fluorescence quantum yield of the dye based on the detected fluorescence intensity. This is no longer possible in cell samples, since the concentration of the probe can significantly vary in various cell compartments, due to different uptake and/or affinity to the fluorophore used. In addition, the quantitative fluorescence intensity measurements in cellular compartments are less reliable, since they suffer

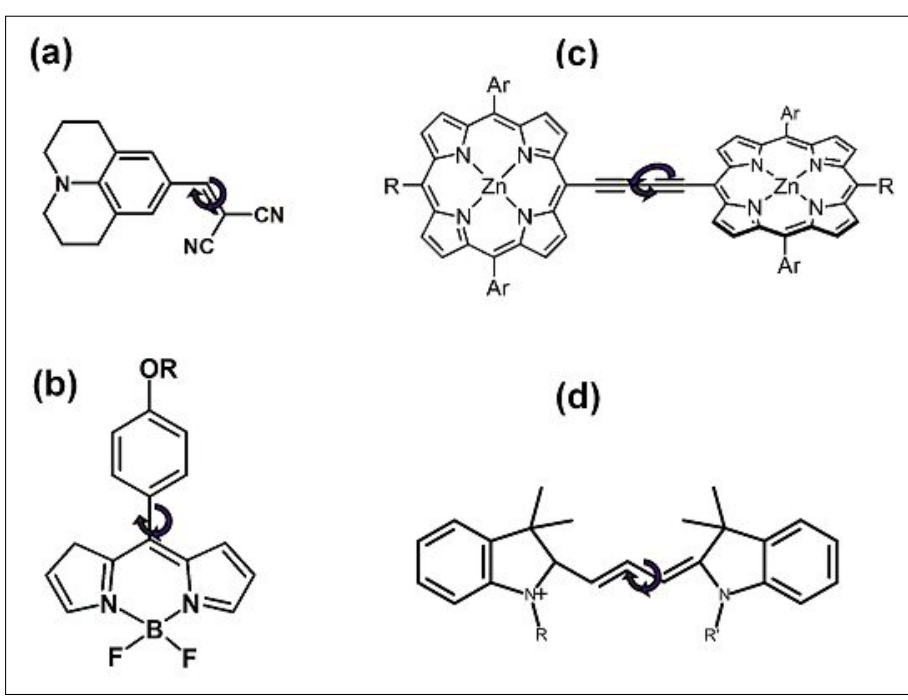

Fig. 1. The structures of molecular rotors that were previously used for various measurements in biological cells: (a) 9-(dicyanovinyl)julolidine (DCVJ) (b) $p$-phenylsubstituted 4,4'-difluoro-4-bora-3a,4adiaza-s-indacene, BODIPY-phenyl- $\mathrm{C}_{12}$; (c) conjugated porphyrin dimer; (d) substituted indocyanine dye (cy3). 
from uncertainties due to inhomogeneities in the optical properties of the medium of cell compartments. In other words, fluorescence intensity changes observed for molecular rotors inside cellular samples can no longer be unequivocally assigned to viscosity changes. The Förster-Hoffman equation in its original form (Eqn. (1)) is rarely applicable to imaging applications within heterogeneous samples, especially, within cells.

We perceived two possible solutions to this problem. Firstly, the ratiometric method can be used, i.e. the conjugation of the molecular rotor to a dye, which is insensitive to viscosity can be utilised for the purposes of internal concentration calibration. Alternatively, it could be useful to determine the dependence of fluorescence lifetime, $\tau_{f}$, on viscosity. Each of these approaches will be discussed in separate sections below.

\section{Fluorescence Lifetime-based Molecular Rotors}

To overcome the problem, associated with the uncertainty of probe concentration within cells, we have designed molecular rotors which can be calibrated using fluorescence lifetime instead of intensity. ${ }^{[8,10 a]}$

According to Eqns (3) and (4), it is clear that the molecular rotor fluorescence lifetime can serve as a good measure of environmental viscosity. Unlike fluorescence intensity, the lifetime does not change as a function of fluorophore concentration. There are only two cases when the concentration of the fluorophore can indeed affect the decay. These are (i) aggregation or dimerisation of fluorophore, which can lead to a species with a separate emission band, or a different fluorescence lifetime, (ii) self-quenching of the excited state of the rotor, leading to a shortening of the observed excited state decay. However, even in the presence of the undesirable processes outlined above, the very nature of the time-resolved fluorescence decay can reveal the presence of interfering secondary photochemical processes affecting the rotor, such as dynamic excited state quenching (e.g. by cellular components residing in the vicinity of the rotor) and environment-induced aggregation. These phenomena typically reveal themselves as additional decay components, resulting in a non-monoexponential overall decay of the excited state. Importantly, these processes can be detected using fluorescence lifetime acquisition, whereas the data on dynamic excited state quenching or aggregation are typically not accessible from fluorescence intensity measurements.

We hypothesised that meso-substi-

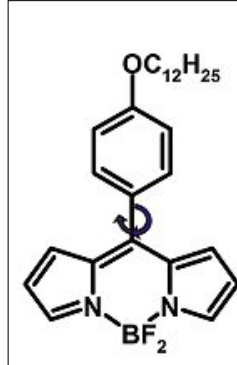

(1)

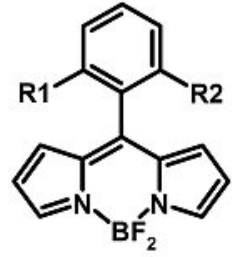

(2a) R1=R2=H

(2b) $\mathrm{R} 1=\mathrm{H}, \mathrm{R} 2=\mathrm{CH}_{3}$ (2c) $\mathrm{R} 1=\mathrm{R} 2=\mathrm{CH}_{3}$

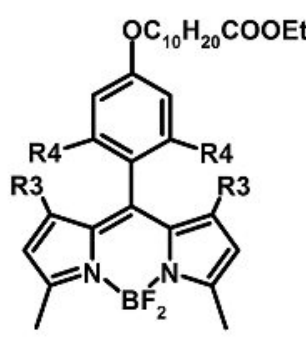

(3a) $\mathrm{R} 3=\mathrm{R} 4=\mathrm{H}$

(3b) R3=H, R4= $\mathrm{CH}_{3}$ (3c) $\mathrm{R} 3=\mathrm{CH}_{3}, \mathrm{R} 4=\mathrm{H}$ (3d) $\mathrm{R} 3=\mathrm{R} 4=\mathrm{CH}_{3}$
Fig. 2. The structures of $p$-substituted BODIPY dyes with various degrees of constraint on rotation of a meso-phenyl group. tuted 4,4'-difluoro-4-bora-3a,4a-diaza- $s$ indacene (1, BODIPY-phenyl- $\mathrm{C}_{12}$, Fig. 2) can act as a molecular rotor based on the previous literature data for similar compounds. For example, for $\mathbf{2 a}$ it was reported that the fluorescence intensity decreases with increasing temperature, when the dark non emissive state becomes accessible and this conclusion was supported by quantum chemistry calculations. ${ }^{[14]}$ The DFT investigation of $\mathbf{2 a}$ has suggested that the 'dark' state is formed as a result of rotation of the phenyl group from the twisted conformation corresponding to the Franck-Condon excited state into the plane of the dipyrrin framework. ${ }^{[14]}$ At the same time, in sterically restricted compounds $\mathbf{2 b}$ and $\mathbf{2 c}$, the free rotation of the phenyl group is impossible. Consistent with our theory, the fluorescent quantum yield increases drastically and becomes very close to unity in these compounds, ${ }^{[15]}$ presumably due to a prevented non-radiative decay. Similarly, in the series of $\mathbf{3 a}-\mathbf{d}$ the fluorescence quantum yield increases from $\mathbf{3 a}<\mathbf{3} \mathbf{b} \approx \mathbf{3} \mathbf{c}<\mathbf{3 d}$ following a decreasing ease of rotation of the meso-phenyl group. ${ }^{[16]}$

The fluorescence measurements of BODIPY-phenyl- $\mathrm{C}_{12}{ }^{[8 \mathrm{a}]}$ made in methanol/glycerol mixtures of different viscosity, Fig. 3, showed that the fluorescence quantum yield increased dramatically with increasing solvent viscosity. The observed increase in fluorescence intensity was con- sistent with the restricted rotation of the phenyl group in solutions of high viscosity, thus preventing relaxation via the population of the dark excited state. In addition, upon viscosity increase from 30 to 1000 $\mathrm{cP}$, the fluorescence lifetime also increased from $(0.7 \pm 0.05)$ to $(3.8 \pm 0.1) \mathrm{ns}$, Fig. $3 b$. The rates of radiative and non-radiative decays were calculated using Eqn. (2) from the measured fluorescence lifetimes and quantum yields. These results demonstrated that for BODIPY-phenyl- $\mathrm{C}_{12}$ the radiative decay rate remained constant in the range of viscosities studied, while the non-radiative decay rate increased with decreasing viscosity by two orders of magnitude. Thus, consistent with the HoffmanFörster theory, BODIPY-phenyl- $\mathrm{C}_{12}$ was shown to be a molecular rotor. Importantly, the fluorescence lifetimes measured in the wide range of viscosities were all in excess of $100 \mathrm{ps}$, falling in the range accessible with time correlated single photon counting (TCSPC). This enabled fluorescence lifetime imaging microscopy (FLIM) of BODIPY-phenyl- $\mathrm{C}_{12}$ to be performed.

Spatially resolved imaging of fluorescence lifetimes (FLIM) became accessible in the late 1990s. In a FLIM experiment the fluorescence decay information across a pixelated field of a fluorescent image is collected and analysed to obtain the value of the fluorescence lifetime in each pixel of the image. ${ }^{[17]}$ This information can be re-

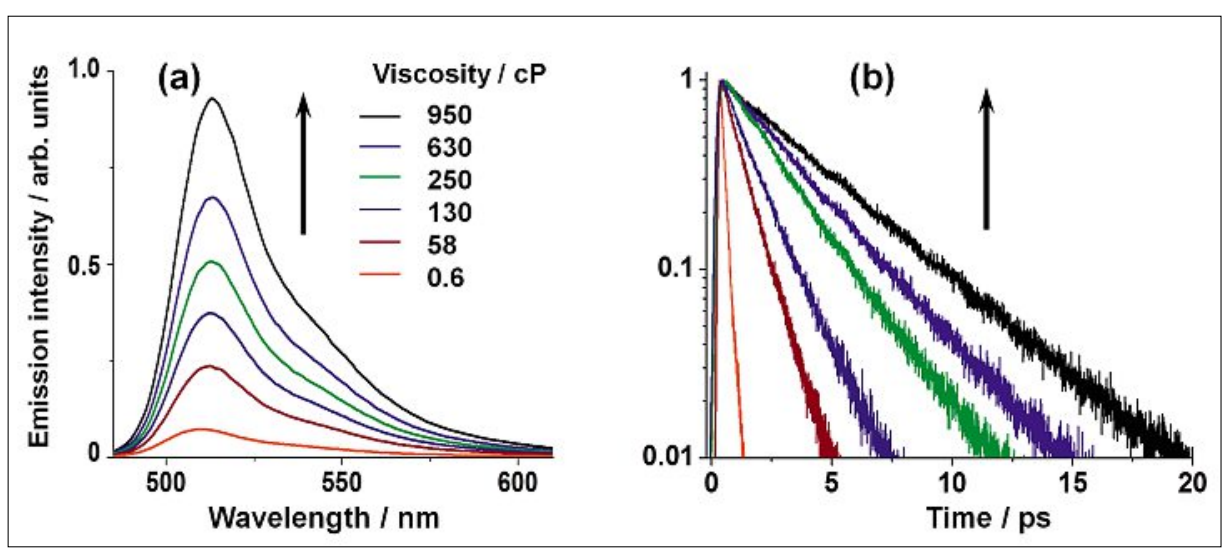

Fig. 3. Fluorescence spectra (a) and decay traces (b) recorded for molecular rotor BODIPYphenyl- $C_{12}$ in methanol/glycerol mixtures of different viscosity. Reprinted with permission from ref. [8a]. Copyright 2008 American Chemical Society. 
corded using either the time- or frequency domain measurements. Time-domain lifetime measurements using time-correlated single photon counting (TCSPC) require the use of a pulsed laser and requires longer signal acquisition times. At the same time, this approach provides a single photon detection capability, which enables precise lifetime determination, ${ }^{[17]}$ This setup is fully compatible with multiphoton excitation from femtosecond lasers. Thus, TCSPC FLIM performed in biological tissues can benefit from reduced scattering and higher spatial resolution offered by multiphoton microscopy. ${ }^{[18]}$

The success of FLIM measurements depends on the availability of molecular rotors with suitable photophysical characteristics. Primarily, their fluorescence lifetime should respond strongly to the changes in the viscosity (large $\alpha$, Eqns (3) and (4)). The lifetimes at various viscosities should also fall within the range accessible with current capabilities of FLIM. In addition, the fluorescence emission needs to be sufficiently bright (appreciable $\Phi_{f}$ ) to allow rapid signal accumulation. The molecular rotors based on meso-phenyl BODIPY structure fit all these requirements. ${ }^{[8,10 a]}$ The longer fluorescence lifetime and higher quantum yield for a given viscosity for this type of rotors present significant advantages over the more commonly used and commercially available maleononitriles and julolidines. ${ }^{[7]}$ The superior fluorescence parameters allow for the successful implementation of BODIPY rotors as microviscosity probes in TCSPC FLIM and, due to the higher observed fluorescence intensities, provides fast image acquisition times. This allowed us to use BODIPY for quantitative viscosity studies in live cells.

For cellular studies we synthesized and studied two meso-phenyl BODIPY derivatives: BODIPY-phenyl- $\mathrm{C}_{12}$ and BODIPYphenyl-farnesyl. ${ }^{[8]}$ Both compounds were identified as fluorescent molecular rotors from the calibration graphs recorded in the methanol/glycerol mixtures of varying viscosity, as shown in Fig. 4. For both dyes the lifetimes recorded at viscosities from $1-1000 \mathrm{cP}$ fell within the accessible range with TCSPC, although the actual lifetime values were slightly different for each dye. The major difference between the two fluorophores was the nature of the tail group, saturated hydrocarbon $\mathrm{C}_{12} \mathrm{H}_{25}$ or farnesyl. The latter is known to present a better structural match to the components of the cellular membranes. Due to this we hypothesised that the farnesyl derivative would partition to the cellular membrane domains better than the $\mathrm{C}_{12}$ derivative. Both fluorophores were hydrophobic and insoluble in water and this made them ideal for imaging microviscosity in the
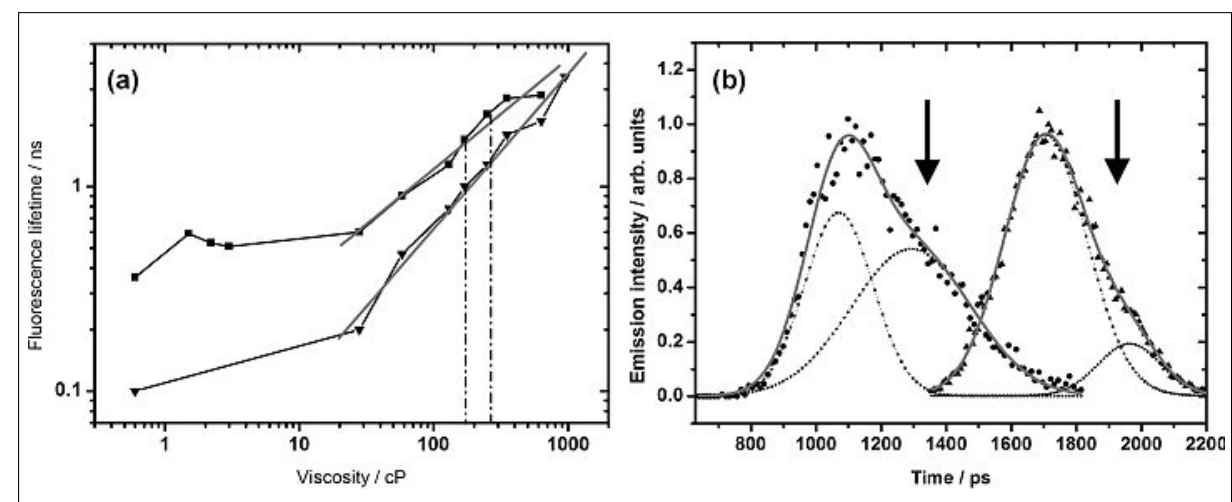

Fig. 4. Fluorescence lifetime data for BODIPY-phenyl- $\mathrm{C}_{12}$ (filled squares) and BODIPY-phenylfarnesyl (filled triangles). (a) Log fluorescence lifetime vs log viscosity; (b) Fluorescence lifetime histograms from FLIM measurements in intracellular environments. The asymmetric distributions can be adequately described and fitted assuming bimodal distribution of the fluorescence lifetimes. The maxima of each lifetime components are shown in (a) by dashed lines. Reprinted with permission from ref. [8b]. Copyright 2009 American Chemical Society.
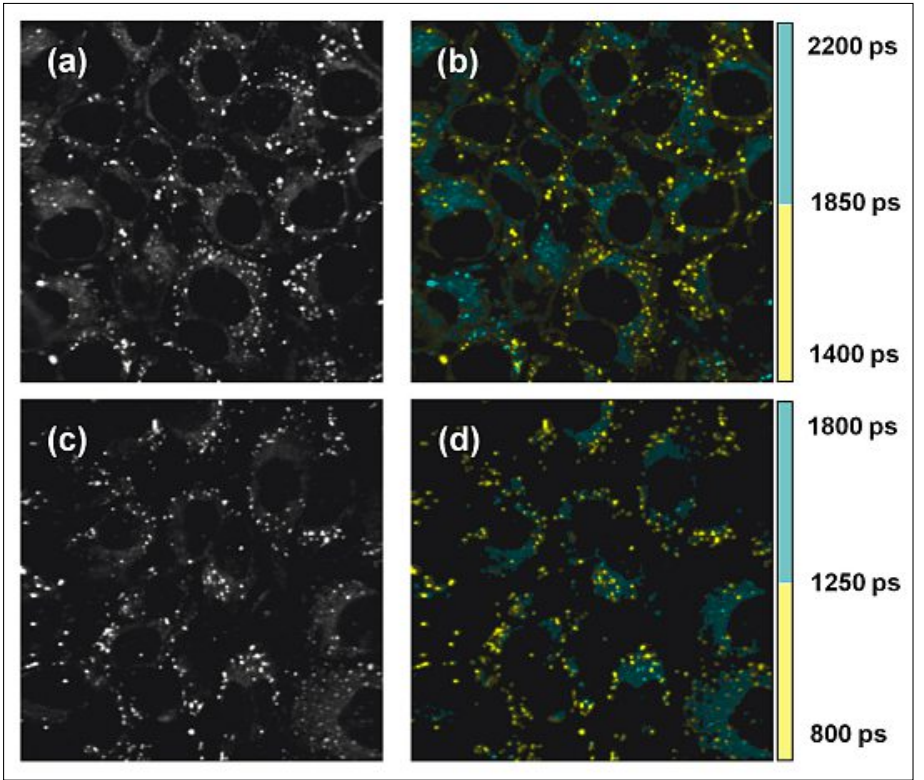

hydrophobic membrane domains within live cells using FLIM.

We separately incubated BODIPYphenyl- $\mathrm{C}_{12}$ and BODIPY-phenyl-farnesyl with SK-OV-3 epithelial adenocarcinoma cells and used FLIM to determine the viscosity. The confocal fluorescence images of the cells are shown in Fig. 5 and showcase the high intracellular uptake of the molecular rotors. The FLIM images were obtained using excitation with a pulsed diode laser at $467 \mathrm{~nm}$ and a narrow lifetime distribution between $1.4 \mathrm{~ns}$ and $2.0 \mathrm{~ns}$ can be seen for BODIPY-phenyl- $\mathrm{C}_{12}$, Fig. 4b. The lifetime distribution for BODIPYphenyl-farnesyl is between 0.8 and $1.6 \mathrm{~ns}$, Fig. 4b. According to the calibration graph for both dyes, Fig. 4a, this range of lifetimes inside the cell corresponds to the same average viscosity of $(160 \pm 40) \mathrm{cP}$.

Importantly, the fluorescence lifetime distributions obtained from SK-OV-3 cells were non-symmetric, and this was particularly obvious for the farnesyl derivative, Fig. 4b. In fact, careful consideration of the images for both dyes, Fig. 5, led to the

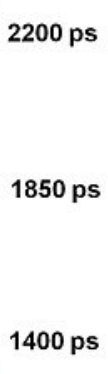

Fig. 5. Fluorescence intensity $(a, c)$ and FLIM images $(b, d)$ for live SK-OV-3 human ovarian carcinoma cells incubated with BODIPY-phenyl- $\mathrm{C}_{12}$ $(a, b)$ and BODIPYphenyl-farnesyl (c, d). The discrete colour scale shows shorter lifetimes in yellow blue. (b) for BODIPYphenyl- $\mathrm{C}_{12}$ : 13501850 ps in yellow and $1850-2200$ ps in blue (d) for BODIPYphenyl-farnesyl: 8001250 ps in yellow and $1250-1800$ ps in blue. Reprinted with permission from ref. [8b]. Copyright 2009 American Chemical Society.

conclusion that two high viscosity domains within SK-OV-3 cells were detected, characterized by apparent viscosities of $160 \pm$ $20 \mathrm{cP}$ and $260 \pm 40 \mathrm{cP}$.

Indeed, most of the dye inside the cells was localised within small vesicle-like domains, characterised by bright punctuate fluorescence pattern and a shorter lifetime corresponding to viscosity of $c a .160 \mathrm{cP}$. However, in addition to the bright punctate distribution, regions of lower fluorescence intensity could be observed within the cell cytosol. These areas were characterised by a longer lifetime, corresponding to viscosity of $c a .260 \mathrm{cP}$. It is very important that every pixel of the image had monoexponential fluorescence decays. Thus, the fluorescence lifetime characterised the viscosity of the unique microenvironment in each point that was 'homogeneous'.

Each lifetime was found in a distinct intracellular domain: shorter lifetimes within and longer lifetimes in 
endosomes (or lipid droplets) characterized by viscosity of $c a$. $160 \mathrm{cP}$, while the longer lifetime was found in other hydrophobic domains with viscosity of $c a .260$ cP. In both cellular domains, independent of the nature of the hydrophobic chain of BODIPY, the measured viscosity values significantly exceeded those expected for the aqueous phase of cellular cytoplasm. These high viscosity values suggested slow diffusion and reaction rates in these hydrophobic cellular microenvironments.

In summary, the fluorescence lifetime maps of BODIPY molecular rotors localised within hydrophobic domains of live cells provided a direct measurement of intracellular viscosity with the spatial resolution of a confocal microscope. These experiments have a level of detail and quantitative information, which was previously unattainable.

\section{Ratiometric Fluorescence Molecular Rotors}

The second approach that allows quantitative determination of viscosity by fluorescence of molecular rotors is based on ratiometric fluorescence detection. In the simplest case, a ratiometric viscosity sensor is constructed to incorporate two independent chromophores. One of the chromophores is not affected by viscosity and is used to determine the concentration of the construct as a whole, whilst the other chromophore acts as a molecular rotor. The ratiometric fluorescence detection of such a probe allows to overcome measurement uncertainties associated with heterogeneous probe distribution, as well as variations in concentration. The other obvious advantage of the ratiometric approach is the much simplified detection setup, which does not require pulsed excitation or timeresolved detection.

One such sensor has been reported by Mark Haidekker and co-workers and used for viscosity measurements in bulk liquids. ${ }^{[19]}$ Due to a lack of biocompatibility, the use of this probe in a biological environment was not possible to date.

The variation of the ratiometric molecular rotor approach was first applied to live cells by Luby-Phelps et al., ${ }^{[20]}$ with the purpose of establishing the viscosity in the aqueous phase of the cellular cytoplasm. This study suggested to use the ratiometric fluorescence detection from the mixture of cyanine dyes cy5 and cy3. Whilst the cy 3 (Fig. 1d) displayed a strong dependence of fluorescence quantum yield on viscosity and acted as a molecular rotor, cy5 did not. Assuming equal uptake of both dyes into the cell, this work has established that the viscosity of the aqueous phase of the cellular cytoplasm does not exceed 6-7 cP. The drawback of this approach was that cy3 and cy 5 were used as a mixture and their equal concentration at the target could not be guaranteed.

We have recently reported a new type of ratiometric molecular rotor, a conjugated butadiyne-linked porphyrin dimer.[21] The dimer works as a molecular rotor, due to the coexistence of two spectroscopically distinct conformations: planar and twisted, with respect to the relative position of the porphyrin units. Each conformer is characterized by distinctive absorption and emission spectra. ${ }^{[21,22]}$ Since rotation around the butadiyne linker is involved in the interconversion between the planar and the twisted fluorescent forms, it was not surprising that the excited state properties of the porphyrin dimer were found to be viscosity dependent.

The emission of the dimer in non-viscous solution was dominated by the lower energy planar conformation, due to the ease of rotation of the porphyrin ring in this environment. Conversely, in a high-viscosity environment the emission of the less stable twisted conformer could be observed, since the rate of the porphyrin ring rotation was slowed down. We established that by using a well defined excitation wavelength the ratiometric response of the rotor (emission intensity of the twisted $v s$ the planar conformations) could be calibrated against

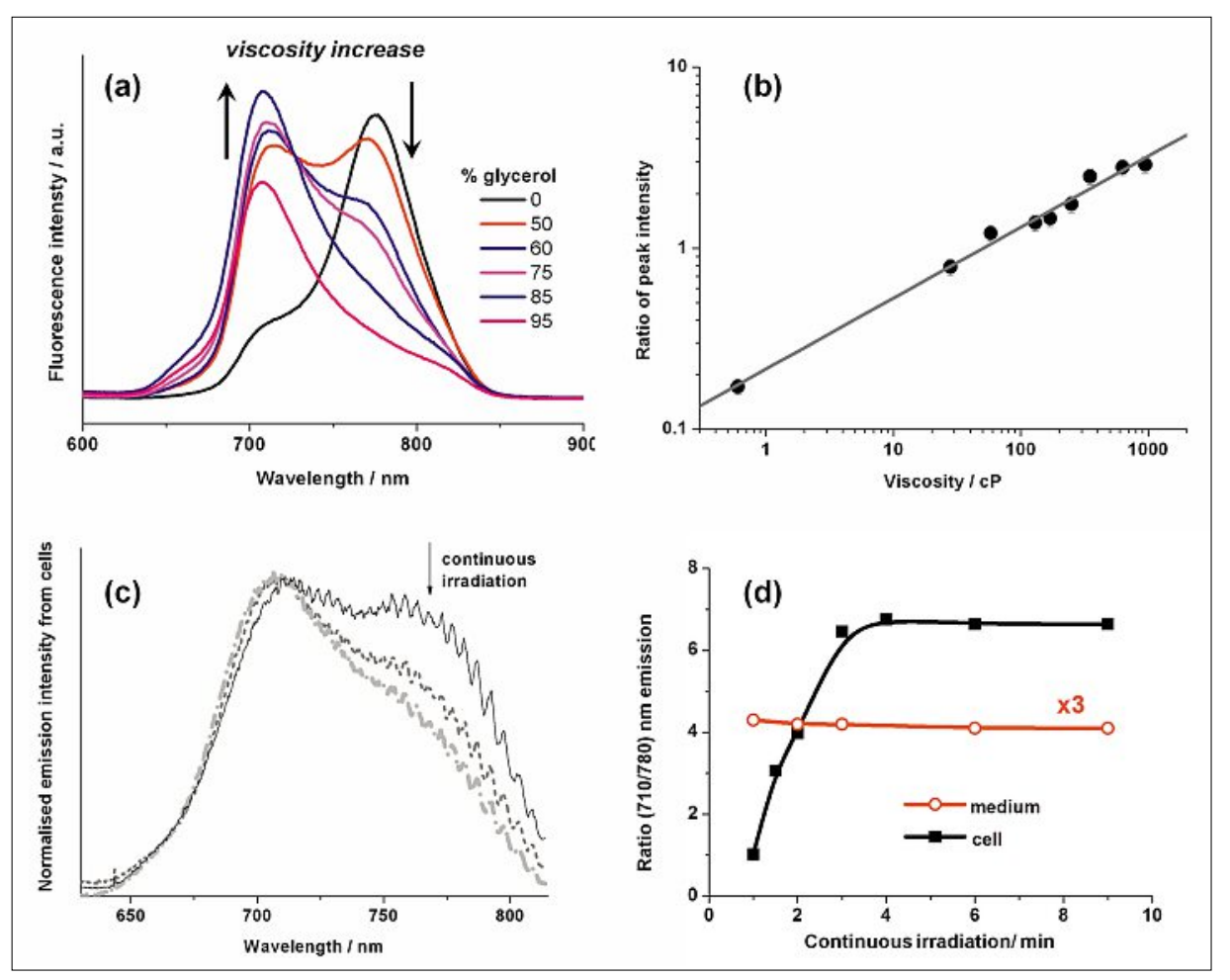

Fig. 6. Fluorescent ratiometric viscosity measurements utilising the conjugated porphyrin dimer. (a) Fluorescence spectra of the dimer, obtained in methanol/glycerol mixtures of different viscosity between $0.6-1000 \mathrm{cP}$; increasing viscosity leads to a higher fluorescence intensity at $710 \mathrm{~nm}$; (b) the fluorescence ratiometric calibration graph of microviscosity; (c) fluorescence spectral measurements in $\mathrm{CHO}$ cells during photodynamic therapy; (d) the intensity ratio of the emission peaks at 710 and $780 \mathrm{~nm}$ versus irradiation time in a cell (black, solid) and in a culture medium (red, hollow). The intracellular intensity ratio increases following irradiation, corresponding to a significant increase in local microviscosity. viscosity according to Eqn. (1), in a large viscosity range between $0.6-1000 \mathrm{cP}$, Fig. 6. We then used the dimer to image viscosity in cells during photoinduced cell death.

\section{Ratiometric Molecular Rotor Measures Viscosity during PDT}

It was recently demonstrated that conjugated porphyrin dimers, such as the rotor shown in Fig. 1c, are very potent photosensitisers for photodynamic therapy (PDT) of cancer, via one- and two-photon excitation. ${ }^{[23]}$ This means that these molecules can be delivered into cells efficiently, are -toxic to cells in the absence of irrairradiation with light.[24] The mechanism of cell kill involves apoptosis or necrosis following production of the cytotoxic short-lived intermediates, 'reactive oxygen species', or ROS. ROS kill malignant cells in the irradiated area, whilst leaving the non-irradiated area untouched, due to the extremely short lifetime and diffusion distance. In a PDT treatment, selective uptake of the drug to the malignant tissue, as well as targeted irradiation of the diseased area with light ensures dual selectivity of the PDT towards cancer versus the normal tissue. [24] diation (negligible 'dark toxicity'), and 
It has been demonstrated in the previous sections that (i) viscosity inside cellular organelles can be very high and (ii) viscosity inside cells is highly heterogeneous. These data are consistent with the literature spectroscopic data from single-point measurements on the diffusion-controlled processes and reactions of short-lived cytotoxic singlet molecular oxygen in single cells. ${ }^{[6,25]}$ We also expected that viscosity in the cell could change as a result of various intracellular processes, for example, cell death. To test this hypothesis we needed to be able to map heterogeneous environment of the cell with sub-micrometer spatial resolution and in real time, on a time scale faster than the cell death process itself. Fluorescence ratiometric imaging with the conjugated porphyrin dimer fulfilled these requirements.

Thus, we have decided to utilise the viscosity-dependent spectroscopic properties of the conjugated porphyrin dimer and apply it simultaneously as a molecular rotor and as a PDT photosensitiser. ${ }^{[21]}$ It is important to note here that ratiometric intensity readings from the dimer can be obtained with low doses of incident light, which are not toxic to cells. At the same time, the irradiation of a conjugated porphyrin dimer inside live cells with high doses of light induces cell death in response to PDT.[23]

The single point fluorescence spectral measurements of the dimer in live unperturbed cells demonstrated unequivocally that the viscosity in the cell compartments around the dimer is very high, $\mathrm{ca} .80 \mathrm{cP}$. Moreover, the domains of different viscosity were visualized using wide-field ratiometric imaging of viscosity. ${ }^{[21]}$ This result emphasised the importance of imaging approach, versus single point acquisition.

Furthermore, the fluorescence spectra of the dimer were recorded following continuous irradiation of the cell, Fig. 6c. These measurements established that the viscosity in the cell increased dramatically as a result of PDT-induced cell death, Fig. 6d. ${ }^{[21]}$ The analysis of the ratiometric fluorescence spectra of the dimer from the necrotic cell subjected to PDT, yielded an unprecedentedly high viscosity value of $360 \mathrm{cP}$.

This increase of the intracellular viscosity as a result of cell death is noteworthy since it manifests the slowing down of the many intracellular processes, which rely on diffusion, such as signalling, transport and bimolecular reactions.

It remains to be established whether the increase of the intracellular viscosity is the cause of cell death or whether this increase is simply a consequence of the oxidative processes that cause cell death. Whichever the case, the ability for fast imaging of intracellular viscosity with molecular rotors opens up exciting possibilities to unravel the role of diffusion in photoinduced cell death and, also, to study the normal cell function.

\section{Verification of Viscosity Values obtained with Molecular Rotor Measurements}

One of the potential problems associated with using molecular rotors in cell measurements is binding of rotors to structures in the intracellular environment or to macromolecules. In the case of binding, the rotation of the functional group(s) responsible for the viscosity-sensitive photophysical behaviour might become restricted. In this case the fluorescence of the rotor might indicate that viscosity is 'high' (i.e. no rotation is possible), however, this value will not reflect the real situation and will not correspond to the high viscosity.

There are several ways to ensure that the longer lifetime of molecular rotors in cells corresponds to real microscopic viscosity, and not to binding and/or rotor interactions with the macromolecular structures inside the cells.

One approach is to complement FLIM and/or ratiometric measurements with anisotropy measurements. The theory behind anisotropy measurements is briefly described below.

Following excitation with linearly polarized light, rotational diffusion of the fluorophore in its excited state results in a depolarization of the fluorescence, which can be monitored. ${ }^{[26]}$ The rate of rotational diffusion changes as a function of viscosity. Thus, monitoring fluorescence anisotropy can provide valuable complementary information on the microviscosity of the environment of the probe. It is particularly useful to monitor the time-resolved fluorescence anisotropy, $r(t)$, which is defined by Eqn. (5):

$$
r(t)=\frac{I_{\|}(t)-G I_{\perp}(t)}{I_{\|}(t)+2 G I_{\perp}(t)}
$$

where $I_{\|}(t)$ and $I_{\perp}(t)$ are the fluorescence intensity decays parallel and perpendicular to the polarization vector of the exciting light. The term $G$ accounts for different transmission and detection efficiencies of the imaging system for parallel and perpendicular polarization. ${ }^{[26]}$

For a spherical molecule, $r(t)$ decays as a single exponential and is related to the rotational correlation time $(\theta)$, Eqn. (6):

$$
r(t)=\left(r_{0}-r_{\infty}\right) \exp \left(-\frac{t}{\theta}\right)+r_{\infty}
$$

where $r_{0}$ is the initial anisotropy and $r_{\infty}$ is the limiting anisotropy which accounts for a restricted rotational mobility. ${ }^{[26]}$

For a spherical molecule in an isotropic medium, $\theta$ is directly proportional to the viscosity $(\eta)$ of the solvent and the molecular hydrodynamic volume $(v)$ of the rotating molecule:

$$
\theta=\left(6 D_{r o t}\right)^{-1}=\frac{\eta v}{k_{b} T}
$$

where $D_{r o t}$ is the rotational diffusion constant, $k_{b}$ is the Boltzmann constant and $T$ is the absolute temperature.

A calibration graph for rotational correlation time $v s$ viscosity can be used to calculate the effective microviscosity. This method, for example, was previously used to obtain a limiting viscosity value of $6 \mathrm{cP}$ in the aqueous phase of cell cytosol.[27]

Importantly, time-resolved anisotropy measurements are able to specifically detect $r_{\infty}$ (which may reflect partial or full binding of the fluorophore) and are, thus, not prone to additional error in viscosity determination. ${ }^{28]}$

We collected fluorescence anisotropy decays from regions of interest within SK-OV-3 cells stained by the meso-phenyl substituted BODIPY molecular rotors. ${ }^{[8]}$ The time-resolved traces were extracted and fitted with a single exponential decay model according to Eqn. (6). The measured rotational correlation time was (590 $\pm 110)$ ps, which corresponds to an average viscosity values of $\sim 60 \mathrm{cP}$. Unfortunately, the time-resolved anisotropy imaging (TR FAIM) of BODIPY rotors did not appear experimentally feasible due to the relatively low intensity available in each polarization channel.

Whilst the viscosity values obtained by time-resolved fluorescence anisotropy measurements $(60 \mathrm{cP})$ were slightly lower than those found by FLIM (160 and 260 $\mathrm{cP})$, both independent methods agreed on the same order of magnitude of viscosity in organelles of SK-OV-3 cells. It is possible that time-resolved fluorescence anisotropy measurements only detected the rotation of the fluorophore as a whole, which is in fact partially embedded in the membrane lipid bilayer. Thus it experienced the degree of a hindered rotational mobility in threedimensional space. Another contributing factor could be that the dye is not spherical, as required by the Stokes-Einstein equation (Eqn. (7)), which could have affected the measured viscosity.

Nevertheless, we could be confident that binding of the rotating unit in a BODIPY rotors did not contribute to the observed molecular rotor signal from cells and the microviscosity value obtained from FLIM measurements was, indeed, real. 
The second approach which allowed us to independently verify the viscosity measurements by the conjugated porphyrin dimer, ${ }^{[21]}$ was based on the observation that the viscosity value in SK-OV-3 cells during the measurement was not static; instead it increased drastically as a result of PDT-induced cell death, as was described in the previous section.

We set up a control experiment ${ }^{[21]}$ where the dimer was exposed to a high protein concentration, similar to that expected within a cell, and irradiated the sample. Our hypothesis was that if the initial viscosity value as detected by the ratiometric signal was due to binding, we would have been able to observe a similar viscosity increase in a model protein mixture, similar to that detected from SK-OV-3 cells.

The experiment clearly demonstrated that the initial ratio of the planar/twisted peaks of the dimer from the protein mixture was considerably lower than that recorded in a cell. In addition, the ratio did not change as a function of prolonged irradiation, as it had done in the cell experiment, Fig. 6d. There is no doubt that the exact content of the protein mix in the cell could not be mimicked like for like in our simple cuvette experiment. However, the fact that no increase in the twisted/planar ratio was detected in this control experiment, confirmed that the initial viscosity value in the cell, as well as the observed viscosity increase during PDT, did not originate from binding of the dimer to intracellular targets.

\section{Conclusions and Outlook}

In the last few years molecular rotors have demonstrated their potential usefulness as new versatile tools for probing and imaging viscosity in biological systems, and their applicability to measurements in clinical studies ${ }^{[29]}$ as well as in fundamental studies of single live cells. ${ }^{[8,20,21,30]}$ There are now several types of molecular rotors available, including malononitriles, BODIPY and porphyrin dimers, which have all been used to measure viscosity within individual domains of live cells. However, quantitative measurements were only possible using the fluorescence lifetime-based rotors or the ratiometric rotors, as described in this review.

Our work has identified that the intracellular domains probed by the hydrophobic molecular rotors are characterised by high viscosity, ranging from $80 \mathrm{cP}$ to 260 cP. Fast viscosity imaging was also possible in the individual domains of live cells, with the resolution of a confocal microscope, in a non-invasive way.

In the future the design of new types of rotors with varied cell localisation will allow a wider range of cellular domains to be investigated. With the ongoing developments in this field, in my laboratory and elsewhere, I believe that molecular rotors provide high potential for contributing new knowledge to the vibrant and fast-developing science of intracellular imaging.

\section{Acknowledgements}

I would like to acknowledge the contribution of my co-workers and collaborators, whose names appear on the publications cited in this review. This work was supported by the UK's Engineerings and Physical Sciences Research Council (EPSRC) in the form of the Life Sciences Interface Fellowship (EP/I003983/1) and the Career Acceleration Fellowship (EP/ E038980/1) to MKK.

Received: January 31, 2012

[1] a) M. Shinitzky, in 'Physiology of membrane fluidity', Ed. M. Shinitzky, CRC Press: Boca Raton, 1984; b) G. Deliconstantinos, V. Villiotou, J. C. Stavrides, Biochem. Pharmacol. 1995, 49, 1589; c) O. Nadiv, M. Shinitzky, H. Manu, D. Hecht, C. T. Roberts Jr., D. LeRoith, Y. Zick, Biochem. J. 1994, 298 (Part 2), 443; d) G. S. Zubenko, U. Kopp, T. Seto, L. L. Firestone, Psychopharmacology (Berlin) 1999, 145,175 ; e) S. J. Singer, G. L. Nicolson, Science 1972, 175, 720.

[2] R. Iino, A. Kusumi, J. Fluorescence 2001, 11, 187.

[3] M. J. Dayel, E. F. Y. Hom, A. S. Verkman, Biophys. J. 1999, 76, 2843.

[4] a) J. A. Dix, A. S. Verkman, Biophys. J. 1990 57, 231; b) K. Fushimi, A. S. Verkman, J. Cell Biol. 1991, 112, 719; c) J. Siegel, K. Suhling, S. Leveque-Fort, S. E. D. Webb, D. M. Davis, D. Phillips, Y. Sabharwal, P. M. W. French, Rev. Sci. Instrum. 2003, 74, 182.

[5] a) J. Korlach, P. Schwille, W. W. Webb, G. W. Feigenson, Proc. Nat. Acad. Sci. USA 1999, 96, 8461 ; b) N. L. Thompson, A. M. Lieto, N. W. Allen, Curr. Opinion. Struct. Biol. 2002, 12, 634.

[6] a) T. Breitenbach, M. K. Kuimova, P. Gbur, S. Hatz, N. B. Schack, B. W. Pedersen, J. D. C. Lambert, L. Poulsen, P. R. Ogilby, Photochem. Photobiol. Sci. 2009, 8, 442; b) M. K. Kuimova, G. Yahioglu, P. R. Ogilby, J. Am. Chem. Soc. 2009, 8b1, 332; c) E. F. F. da Silva, B. W. Pedersen, T. Breitenbach, R. Toftegaard, M. K. Kuimova, L. G. Arnaut, P. R. Ogilby, J. Phys. Chem. B 2011, 116, 445.

[7] a) M. A. Haidekker, E. A. Theodorakis, Org. Biomol. Chem. 2007, 5, 1669

[8] a) M. K. Kuimova, G. Yahioglu, J. A. Levitt, K. Suhling, J. Am. Chem. Soc. 2008, 130, 6672; b) J. A. Levitt, M. K. Kuimova, G. Yahioglu, P.-H. Chung, K. Suhling, D. Phillips, J. Phys. Chem. C 2009, 113, 11634.

[9] a) C. E. Kung, J. K. Reed, Biochemistry 1986, 25, 6114; b) C. E. Kung, J. K. Reed, Biochemistry 1989, 28, 6678; c) S. Sawada, T. Iio, Y. Hayashi, S. Takahashi, Anal. Biochemistry 1992, 204, 110.

[10] a) G. Hungerford, A. Allison, D. McLoskey, M. K. Kuimova, G. Yahioglu, K. Suhling, $J$. Phys. Chem. B 2009, 113, 12067; b) A. Rei, G. Hungerford, M. I. C. Ferreira, J. Phys. Chem. B 2008, 112, 8832 .

[11] M. A. Haidekker, C. R. White, J. A. Frangos, J.
Biomech. Eng.-Trans. Asme 2001, 123, 455.

[12] T. Förster, G. Hoffmann, Z. Phys. Chem.Frankfurt 1971, 75, 63.

[13] a) M. Kaschke, J. Kleinschmidt, B. Wilhelmi, Chem. Phys. Lett. 1984, 106, 428; b) B. Wilhelmi, Chem. Phys. 1982, 66, 351.

[14] F. R. Li, S. I. Yang, Y. Z. Ciringh, J. Seth, C. H. Martin, D. L. Singh, D. H. Kim, R. R. Birge D. F. Bocian, D. Holten, J. S. Lindsey, J. Am. Chem. Soc. 1998, 120, 10001

[15] H. L. Kee, C. Kirmaier, L. H. Yu, P. Thamyongkit, W. J. Youngblood, M. E. Calder, L. Ramos, B. C. Noll, D. F. Bocian, W. R. Scheidt, R. R. Birge, J. S. Lindsey, D. Holten, J. Phys. Chem. B 2005, 109, 20433.

[16] K. Yamada, T. Toyota, K. Takakura, M Ishimaru, T. Sugawara, New J. Chem. 2001, 25, 667.

[17] K. Suhling, P. M. W. French, D. Phillips, Photochem. Photobiol. Sci. 2005, 4, 13

[18] F. Helmchen, W. Denk, Nat. Methods 2005, 2, 932.

[19] M. A. Haidekker, T. P. Brady, D. Lichlyter, E. A Theodorakis, J. Am. Chem. Soc. 2006, 128, 398

[20] K. Luby-Phelps, S. Mujumdar, R. B. Mujumdar L. A. Ernst, W. Galbraith, A. S. Waggoner, Biophys. J. 1993, 65, 236.

[21] M. K. Kuimova, S. W. Botchway, A. W. Parker, M. Balaz, H. A. Collins, H. L. Anderson, K. Suhling, P. R. Ogilby, Nature Chem. 2009, 1 , 69.

[22] M. K. Kuimova, M. Balaz, H. L. Anderson, P. R. Ogilby, J. Am. Chem. Soc. 2009, 131, 7948.

[23] a) H. A. Collins, M. Khurana, E. H. Moriyama, A. Mariampillai, E. Dahlstedt, M. Balaz, M. K. Kuimova, M. Drobizhev, V. X. D. Yang, D. Phillips, A. Rebane, B. C. Wilson, H. L. Anderson, Nat. Photonics 2008, 2, 420; b) E. Dahlstedt, H. A. Collins, M. Balaz, M. K. Kuimova, M. Khurana, B. C. Wilson, D. Phillips, H. L. Anderson, Org. Biomol. Chem. 2009, 7, 897; c) M. K. Kuimova, H. A. Collins, M. Balaz, E. Dahlstedt, J. A. Levitt, N. Sergent, K. Suhling, M. Drobizhev, N. S. Makarov, A Rebane, H. L. Anderson, D. Phillips, Org. Biomol. Chem. 2009, 7, 889; d) M. K. Kuimova, M. Hoffmann, M. U. Winters, M. Eng, M. Balaz, I. P. Clark, H. A. Collins, S. M. Tavender, C. J. Wilson, B. Albinsson, H. L. Anderson, A W. Parker, D. Phillips, Photochem. Photobiol. Sci. 2007, 6, 675; e) M. Balaz, H. A. Collins, E. Dahlstedt, H. L. Anderson, Org. Biomol. Chem. 2009, 7, 874

[24] R. Bonnett, 'Chemical Aspects of Photodynamic Therapy', Gordon and Breach Science Publishers, 2000.

[25] S. Hatz, L. Poulsen, P. R. Ogilby, Photochem. Photobiol. 2008, 84, 1284.

[26] a) B. Valeur, 'Molecular Fluorescence', WileyVCH, Weinheim, 2002; b) J. R. Lakowicz 'Principles of Fluorescence Spectroscopy', 3rd ed., Springer: New York, 2006.

[27] K. Suhling, J. Siegel, P. M. P. Lanigan, S. Leveque-Fort, S. E. D. Webb, D. Phillips, D. M. Davis, P. M. W. French, Opt. Lett. 2004, 29 , 584

[28] J. A. Levitt, P.-H. Chung, M. K. Kuimova, G. Yahioglu, Y. Wang, J. Qu, K. Suhling, ChemPhysChem 2011, 12, 662 .

[29] a) W. J. Akers, J. M. Cupps, M. A. Haidekker, Biorheology 2006, 43, 693; b) M. A. Haidekker, A. G. Tsai, T. Brady, H. Y. Stevens, J. A Frangos, E. Theodorakis, M. Intaglietta, Am. J. Physiol.-Heart and Circulatory Physiology 2002, 282, H1609.

[30] M. A. Haidekker, N. L'Heureux, J. A. Frangos, Am. J. Physiol. - Heart and Circulatory Physiology 2000, 278, H1401. 